\title{
Diagnostics de crise
}

Rôle textuel de la dislocation à gauche dans la prose philosophique de Peter Sloterdijk

Krisendiagnosen - die textuelle Rolle der Linksversetzung in philosophischen Texten Peter Sloterdijks

Diagnosis of Crisis - the Left-Dislocations in Philosophical Texts by Peter Sloterdijk

\section{Laetitia Faivre}

\section{OpenEdition}

\section{Journals}

Édition électronique

URL : http://journals.openedition.org/ceg/2358

DOI : 10.4000/ceg.2358

ISSN : 2605-8359

\section{Éditeur}

Presses Universitaires de Provence

Édition imprimée

Date de publication : 26 octobre 2017

Pagination : 113-126

ISBN : 979-10-320-0129-5

ISSN : 0751-4239

\section{Référence électronique}

Laetitia Faivre, «Diagnostics de crise », Cahiers d'Études Germaniques [En ligne], 73 | 2017, mis en ligne le 26 avril 2019, consulté le 23 novembre 2020. URL : http://journals.openedition.org/ceg/2358 ; DOI : https://doi.org/10.4000/ceg.2358 


\title{
Diagnostics de crise \\ Rôle textuel de la dislocation à gauche dans la prose philosophique de Peter Sloterdijk
}

\author{
Laetitia FAIVRE \\ Cité scolaire Roger Frison-Roche, Chamonix
}

\section{Introduction}

Le discours philosophique contemporain a très souvent affaire avec un diagnostic de la société actuelle, qu'il examine et qui nourrit sa réflexion. Il est de fait souvent suscité par une situation de crise dont il va proposer une lecture, identifier les symptômes. Mais il peut également la précéder et la faire advenir, la montrer du doigt ou même la construire par le discours. Si l'analyse est historiographique, si elle s'inscrit dans le temps pour chercher des causes profondes, elle a aussi tendance à chercher les points de rupture, les phases "critiques", à poser les « avant » et les « après ». Qu'elle soit origine, conséquence ou même méthode, la crise ou la mise en crise participe en soi du geste philosophique.

Le philosophe et essayiste allemand Peter Sloterdijk ne dément pas cette affirmation, lorsqu'il affirme en parlant des écrivains d'aujourd'hui et de lui-même qu'ils sont des « individus qui ont à l'oreille les détonations de [leur] temps » (Sloterdijk 2003: 12). II dit également: "Les humains sont des créatures qui, dès qu'elles commencent à penser, sont en quelque sorte prises en otage par les grands thèmes. Dès que nous ouvrons notre cerveau, nous découvrons que nous sommes devenus les otages de problèmes qui nous entraînent quelque part ailleurs » (Ibid.). Cette métaphore de la prise en otage confirme ce rôle moteur de la crise pour la pensée.

En tant que linguiste, je me suis intéressée de plus près à l'agencement du texte en le considérant à travers le prisme de cette idée de crise, si déterminante dans la prose sloterdijkienne. Mon attention s'est focalisée sur le phénomène des dislocations à gauche (DG), auxquelles l'auteur a souvent recours. Par DG, j'entends avec Mathieu Avanzi « les énoncés dans lesquels un argument du verbe est donné deux fois ", "[u]ne fois sous une forme pleine, dans la périphérie gauche de l'énoncé [Rizzi 1997'], et une autre fois sous la forme d'un pronom

1. Cité par Avanzi (2011). 
co-référent, contenu dans la construction verbale adjacente » (Avanzi 2011 [en ligne]). J'introduis une modification: la deuxième apparition de l'argument du verbe peut se faire sous la forme d'un syntagme nominal démonstratif (anaphore résomptive), même si cette configuration va questionner l'autonomie du segment disloqué (la dislocation n'existe plus que de manière ténue à l'échelle du texte) en même temps qu'elle va nous donner des informations sur la structure disloquée. Ne seront pas retenues ici les définitions qui envisagent la construction disloquée en termes de déplacement en tête de phrase d'un syntagme, par contraste avec une structure canonique. La dislocation ne déplace pas, elle configure différemment l'énoncé. L'élément en tête fait se dérouler autrement l'ordre des mots.

Si les DG sont extrêmement étudiées à l'oral, où elles sont effectivement fréquentes, elles restent peu et / ou mal étudiées à l'écrit. Elles ont ainsi souvent tendance à être analysées, en allemand et en français, comme une résurgence d'oralité dans le texte écrit (Blasco-Dulbecco 1999, Schanen / Confais 2001), mais les fonctions qui leur sont associées à l'oral occultent partiellement leur véritable rôle textuel et argumentatif. La question au centre du présent article est la suivante: cette syntaxe caractéristique de la DG, si particulière, disloquée, disjointe, peut-elle être mise en lien avec la notion de diagnostic de crise dans le genre philosophique, et la servir textuellement?

Les exemples étudiés sont tirés de deux ouvrages de Peter Sloterdijk: Die Verachtung der Massen (2000) et Sphären II (1999). II est en effet difficile, même par une lecture cursive, de ne pas repérer les très nombreuses DG dans ses textes, écrits dans une langue souvent décriée, que lui-même sait et veut particulière ${ }^{2}$. Seront étudiées ici quelques-unes des occurrences les plus représentatives.

\section{La DG dans l'organisation textuelle}

\section{Rôle signalétique}

La DG joue un rôle particulier dans l'organisation du texte. On peut mentionner en premier un rôle d'« étiquetage».

Cet effet signalétique est accentué (visuellement) chez Sloterdijk par I'utilisation quasi systématique du tiret demi-cadratin:

2. « Si j'en crois à la lecture que je fais de mes textes, je reste dans l'espace élargi de la prose philosophique, et donc dans le domaine des concepts, même s'il s'y ajoute, plus que d'ordinaire, des éléments imagés, métaphoriques, sonores. J'estime de toute façon que le discours évocateur est un élément légitime de la philosophie. » (2003: 182); "La philosophie a toutes les raisons de regarder de plus près la richesse rationnelle virtuelle des langages poétiques et de rendre la connaissance du discours poétique féconde pour la constitution théorique de modèles. » (2003: 184). 
(1) Der wesentliche Unterschied - das ist die Spur des ancien régime, die es zu auszulöschen gilt; die wesentliche Gleichheit - sie weist der kommenden respublica die Bahn. (Sloterdijk $2000: 71)^{3}$

L'élément disloqué a une fonction de rappel dans le système du texte, comme pour un index, et contribue à une identification rapide de concepts ou situations jugés critiques. Ici, les deux groupes nominaux se détachent du texte et se répondent. Ils évoquent, de manière épurée, la transition d'un monde à un autre, le passage de l'aristocratie à la démocratie égalitaire au centre de la réflexion du philosophe sur la société de masse.

\section{Rôle cadratif}

La DG garantit par ailleurs une chaîne référentielle claire. L'exemple suivant montre la façon dont les deux DG guident la lecture, presque aussi clairement que le feraient un a) et un b):

(2) Daß es sich um ein Gelehrtengespräch handelt, das zeigen die philosophentypischen Bärte der Teilnehmer und die Buchrollen in den Händen einiger Diskutanten. Und daß es sich um einen Ausnahmezustand des Denkens handelt: das geht hervor aus der Gegenwart eines Objekts, von dem mit Sicherheit angenommen werden muß, daß es den Grund der Versammlung und das Motiv des gemeinsamen Enthusiasmus bildet. (Sloterdijk 1999: 14)

On peut faire l'hypothèse que la référence posée étant complexe, le scripteur procède à une simplification de l'encodage. Cette hypothèse s'appuie sur les travaux de Laure Gardelle (2010) pour l'anglais:

[L]e constituant disloqué crée une unité informationnelle qui pose la référence. L'effort d'encodage est ensuite moindre par rapport à une structure non disloquée, car la référence est déjà établie au moment où la prédication est énoncée. (Gardelle 2010 [en ligne])

Si l'on restitue pour (2) le cotexte gauche, on s'aperçoit qu'il s'agit d'une question, à laquelle il est répondu en deux temps. Cela confirme une fonction résumante (qui produit une forme de soulagement au niveau mémoriel pour le lecteur) des deux segments disloqués [a] et [b].

(2a) Wie ist es möglich, dergleichen aus diesem Bild zu lesen? [a] Daß es sich um ein Gelehrtengespräch handelt, das zeigen die philosophentypischen Bärte der Teilnehmer und die Buchrollen in den Händen einiger Diskutanten. Und [b] daß es sich um einen Ausnahmezustand des Denkens handelt: das geht hervor aus der Gegenwart eines Objekts, von dem mit Sicherheit angenommen werden muß, daß es den Grund der Versammlung und das Motiv des gemeinsamen Enthusiasmus bildet. (Sloterdijk 1999: 14)

3. Dans les exemples étudiés, l'élément disloqué à gauche et l'élément de reprise dans l'énoncé d'accueil sont en italique. 
La réponse redéveloppe " dergleichen », orienté vers l'amont ${ }^{4}$ et l'aval du texte, à la fois anaphorique et cataphorique, et cette manière d'écrire est à mettre en lien avec la rhétorique de la démonstration.

Cette opération en deux temps en laquelle consiste la dislocation permet l'élaboration d'une prédication complexe, à plusieurs niveaux. Dans un premier temps, le thème est posé en périphérie gauche. Puis dans un second temps, une prédication lui est appliquée dans l'énoncé d'accueil:

(3) Denn Optimist sein - das ist keine Charakter- und Stimmungsfrage mehr; es bedeutet jetzt nichts anderes, als sich im Denken den besten Gründen aussetzen, um von ihnen enteignet, aufgeheitert, gesteigert zu werden. (Sloterdijk 1999: 27)

La valeur cadrative du segment antéposé est, dans cet exemple, évidente, puisqu'il est repris dans deux énoncés consécutifs, une première fois par « das ", une seconde par « es » (cf. Charolles / Péry-Woodley 2005), sa portée s'étend aux deux phrases (au sens grammatical du terme), comme le montre le test de remplacement:

(3a) Denn [Optimist sein] ist keine Charakter- und Stimmungsfrage mehr; [Optimist sein] bedeutet jetzt nichts anderes, als sich im Denken den besten Gründen aussetzen, um von ihnen enteignet, aufgeheitert, gesteigert zu werden.

La définition de l'optimisme est négative, puis positive. On constate ce rôle organisateur de l'expression disloquée au sein du texte et son utilité presque didactique pour la lecture. "Voici de quoi je parle ", puis " voici ce que j'en dis ». Ces fonctions signalétiques et cadratives confirment l'idée selon laquelle la place la plus à gauche serait la place idéale du bilan et du diagnostic. Cet « état d'urgence » dont il est question en (2), ce n'est rien moins que la naissance de la philosophie avec la question ontologique. Dans son prologue de Sphären II, Sloterdijk fait l'analyse d'une scène qu'il présente comme un moment crucial de la pensée. Tout le prologue est construit sur une mosaïque que l'on peut contempler au Musée national de Naples et qui daterait d'environ un siècle avant J.-C. Ce qui l'intéresse, c'est le motif de la boule (« Kugel »), motif majeur de ce deuxième tome de la trilogie des Sphères (I Bulles, II Globes, III Écumes). $\mathrm{Au}$ centre de la mosaïque, on observe une boule bleue claire (une sphère, un globe) recouverte d'un réseau de lignes rougeâtres qui s'entrecroisent. Accordés et fascinés autour de la boule, sept sages de la Grèce Antique. Je vais procéder maintenant à une analyse en deux temps, par deux " coups de loupe » sur cette tournure syntaxique, en regardant d'abord d'un peu plus près la partie disloquée, puis la reprise dans l'énoncé d'accueil. Cette dissociation dans l'analyse n'a qu'une visée pratique et ne doit surtout pas conduire à une vision binaire ou

4. Fin du paragraphe qui précède: «Der fruchtbare Augenblick in dieser dotta conversazione ist gekommen. Das Wortemachen hat sich ins Denken verwandelt; aus dem vergeblichen Gerede aufsteigend, beginnen weltergreifende Ideen ihren Flug. Eine Evidenz wie keine vor ihr zieht die Intelligenz der Anwesenden in ihren Bann » (Sloterdijk 1999: 14). 
séparée de la structure. Ce détour est néanmoins nécessaire pour comprendre certains aspects de son fonctionnement global.

\section{« Un thème qui toujours déjà prédique » (Siblot 1998)}

Pour Gardelle, le constituant disloqué à gauche se situe hors prédication:

[...] la dislocation opère une dissociation du rôle et de la référence (et par conséquent [elle peut] avoir une fonction de simplification du traitement de l'information): le constituant disloqué n'occupe pas de fonction syntaxique dans la proposition. Pour cette raison, d'ailleurs, un pronom anaphorique doit être présent dans la proposition: le rôle du référent dans le procès doit y être indiqué. Cette caractéristique explique également pourquoi le constituant disloqué ne peut être focus (au sens de porteur de la charge informative nouvelle de l'énoncé): un focus est apporté par une prédication, alors que le rôle du constituant disloqué est précisément de poser une référence, donc de se trouver hors prédication. [souligné par LF] (Gardelle 2010 [en ligne])

Il faut préciser que dans toutes les dislocations analysées par Gardelle en anglais, le constituant disloqué est un syntagme nominal, naturellement référentiel. Or dans le corpus étudié, les éléments disloqués présentent très souvent une structure complexe, qu'il est difficile voire impossible - on le verra en (6) - de penser « hors prédication », même pour les syntagmes nominaux ${ }^{5}$. Sloterdijk disloque souvent à gauche un groupe nominal complexe [adjectif + nom (+ expansions à droite)], une expression verbale infinitive [verbe non conjugué + compléments], ou encore une phrase entière [sujet + verbe conjugué + complément, entre guillemets ou non]. Ce dernier cas de figure, aux confins de la dislocation (niveau rectionnel) et d'une nouvelle entité syntaxique, s'avère en fait très éclairant pour mettre en lumière l'autonomie du segment disloqué. Il est également remarquable que la dislocation soit, dans la majorité des occurrences, nettement marquée par un tiret demi-cadratin, qui interrompt visuellement la continuité avec l'énoncé d'accueil et qui fait aussi que l'on s'y attarde un peu plus.

\section{Groupe nominal}

Effectivement, ce cas de figure correspond plutôt bien à ce qui a été décrit dans la première partie de cet article: la référence est posée, puis on lui prédique quelque chose. Cependant, l'association du substantif et de l'adjectif est souvent déjà hautement prédicative.

Dans (4), il s'agit d'une reprise d'un contenu informationnel déjà mentionné dans le texte, mais ce contenu est problématisé. Sloterdijk montre du doigt un oxymore - "recherche vénérante » (traduit par LF) -, oxymore qu'il explicite dans l'énoncé d'accueil par « la contradiction dans l'adjectif » (traduit par LF):

5. À moins de postuler un effet de nominalisation de la DG (« mécanisme de transgression catégorielle », cf. Authier-Revuz 2002). 
(4) Verehrende Forschung - der Widerspruch im Beiwort klärt sich auf, sobald man sich vergegenwärtigt, daß die Kugel für die Alten, insbesondere nach der platonischen Reform der sprüchekündenden Weisheit zur argumentierenden Philosophie, als Emblem des göttlichen Seienden im ganzen gegolten hatte - Symbol für jenes Umgreifende oder UmSein, periéchon, das alle physischen und geistigen Gattungen des Seienden in sich faßt und das somit auch die Intelligenzen durchwirkt, die sich in diesem Augenblick über die allmächtige Kugel beugen. (Sloterdijk 1999: 15)

La collision sémantique entre le substantif et l'adjectif souligne le rôle prédicatif de ce dernier. Le placement en tête permet de laisser résonner la figure pour ellemême. Sloterdijk établit le diagnostic concis mais efficace d'une situation qu'il souhaite absolument présenter comme un moment charnière de la philosophie. II l'explicite quelques lignes plus loin. Cette scène est selon lui un point de rencontre inédit et non gênant entre culte et discours, entre vénération et logos; les analyses, les mesures et les preuves viennent remplacer les prières et les invocations pour s'élever vers la divinité (transcendance). L'auteur insiste d'ailleurs, en utilisant le même tour, pour le même objet de discours, avec un groupe nominal disloqué extrêmement complexe, comme on le voit dans (5):

(5) Sieben Gelehrte angesichts einer gebänderten Kugel, bärtige Herren in einer Heiterkeit, deren Grund kein Außenstehender errät, aus der Stadt entrückt, einer subtilen Dissidenz hingegeben, durch gemeinsame logische Intuitionen eingeschworen auf eine unendliche Frage - das ist die Urszene des akademischen Pazifismus. (Sloterdijk 1999: 19)

L'association [nom commun + expansion à droite + appositions] fait l'effet d'un arrêt sur image, et on ne peut si bien dire car le texte de Sloterdijk dans sa trilogie des Sphères est illustré: l'élément disloqué est une traduction en mots de l'image qu'il commente dans son prologue, une scène qu'il tient absolument à poser comme originelle ("Urszene »). Le segment antéposé est cerné de part et d'autre, ce qui souligne son importance:

(5a) Kein Intellektueller dürfte je diese Situation vergessen: Sieben Gelehrte angesichts einer gebänderten Kugel, bärtige Herren in einer Heiterkeit, deren Grund kein Außenstehender errät, aus der Stadt entrückt, einer subtilen Dissidenz hingegeben, durch gemeinsame logische Intuitionen eingeschworen auf eine unendliche Frage - das ist die Urszene des akademischen Pazifismus. (Sloterdijk 1999: 19)

À deux reprises, dans le Prologue qui ouvre le tome II des Sphères, Sloterdijk utilise la dislocation pour saisir ou ressaisir cette scène primitive, fondamentale, qu'il s'agit ensuite de commenter. La dislocation du groupe nominal est sans doute le cas le plus prototypique et le plus aisément descriptible. Le nom est de fait la catégorie a priori la plus à même de poser une référence. L'espace libéré à la périphérie gauche de la phrase est plastique et peut accueillir, on le voit, des éléments de taille conséquente. C'est un moment d'harmonie, il est vrai, ainsi que l'exprime l'idée de « pacifisme académique ». La pensée ne connaît pas de frontières, elle est mondiale. Les philosophes concourent tous au savoir, à la recherche de la vérité. La recherche pure de la vérité, en tant qu'elle est une, serait exempte du conflit d'intérêts. Cela étant dit, il s'agit bel et bien d'une configuration axiomatique nouvelle. La crise mise en exergue par la DG serait ici 
à comprendre comme transition, sans qu'on y associe forcément une douleur, un mal-être (cf. Ricoeur 1988, modèle épistémologique de la crise, I. 4. ${ }^{6}$ ).

\section{Adjectif}

L'adjectif est une catégorie de mot incidente à un autre terme: il est régi soit par un nom (épithète ou apposé même si le lien syntaxique est, dans l'apposition, beaucoup plus lâche), soit par un verbe (attribut, adverbe). En (6), l'adjectif " carlylisch », caractérisé à gauche par un adjectif à valeur adverbiale qui indique une variation de degré, apparaît étonnamment seul, c'est-à-dire non incident à un nom ou à un verbe:

(6) Absolut carlylisch: von diesem Prädikat ist das System der mediatisierten Massenkultur insgesamt betroffen. (Sloterdijk 2000: 23)

Sloterdijk effectue ici une dislocation de la prédication. Une paraphrase de (6) met en évidence la nature rhématique de l'élément disloqué, également explicitée par le terme de reprise «Prädikat » (prédicat) dans l'énoncé d'accueil:

(6a) Das gesamte System der mediatisierten Massenkultur ist absolut carlylisch.

Dans le texte, l'adjectif « carlylisch », création lexicale formée à partir du nom de l'idéologue anglo-écossais Thomas Carlyle (1795-1881), apparaît d'abord dans une citation du sociologue Robert Michels, où il sert à caractériser le fascisme. Carlyle a théorisé la fonction historique et politique du culte de la personnalité. La nature rhématique de l'adjectif doit toutefois être relativisée. L'opération de prédication n'a lieu de façon effective que dans l'énoncé d'accueil, par le biais de l'expression de reprise, complément d'agent, alors qu'elle n'est que virtuelle à l'initiale. En revanche, l'adjectif est bel et bien convoqué comme diagnostic, apposé ensuite à la culture de masse.

\section{Groupe infinitif}

Les éléments détachés sont souvent des expressions à l'infinitif. On associe souvent l'infinitif à une généralisation, une valeur de vérité générale, quelque peu péremptoire. On sort du temps. Cela escamote en partie leur dimension résumante, très efficace en texte (allègement de l'effort mémoriel). Bordas note pour le français que « le détachement rend précisément l'usage de l'infinitif sujet

6. «On retrouve, dans ce modèle épistémologique, quelques-uns des traits des modèles précédents [crise médicale, crise personnelle, crise politique]: d'abord la discontinuité temporelle, ensuite l'idée d'une alternance d'états d'équilibre et de phases de déséquilibre, enfin l'idée d'une complexification croissante du savoir opérée à travers une série de bonds qualitatifs. Ici aussi, la crise est transitoire. On ne la connaît qu'après coup du point de vue de la configuration axiomatique nouvelle. Toutefois, aucune idée de pathologie, de souffrance ou de malaise n'est attachée à ce modèle de crise, à moins que l'on n'appelle ainsi le désarroi intellectuel dans lequel séjournent les tenants de la synthèse dépassée. » (Ricoeur 1988: 5). 
beaucoup plus aisé que dans des phrases nucléaires » (1999: 223), c'est-à-dire dans des phrases déclaratives simples. Cette remarque vaut aussi pour l'allemand.

(7) Sein und Zeit zu begreifen und die Konstellation zu durchleuchten, die beide miteinander bilden - darum und um nichts anderes ist es diesem wortreich dunklen Gewerbe zu tun. (Sloterdijk 1999: 22)

(8) Die Fülle an den Anfang setzen, das wirft auf alles, was der Fall ist, ein aristokratisches Licht. (Sloterdijk 1999: 29)

(9) Die kryptische Identität von Christologie und Kugelmetaphysik auszuarbeiten: dies war in tiefenstruktureller Sicht das Programm der christlichen Geistesgeschichte. (Sloterdijk 1999: 121)

En (7), Peter Sloterdijk cite indirectement l'ouvrage de Martin Heidegger Sein und Zeit (1927) qui reprenait à nouveaux frais la question du sens de l'être, longtemps laissée de côté par la philosophie. Sur la mosaïque analysée, on voit en effet une sphère, au centre, ainsi qu'une horloge, derrière les penseurs, en haut de la mosaïque. Cette configuration est la clé de l'activité philosophique. Sloterdijk, par cette lecture, semble dire que tout était déjà là.

En (8), Sloterdijk montre que, à l'inverse de la psychanalyse - qu'il désavoue dans la plupart de ses écrits - qui pense le rapport au réel en termes de manque et de castration, la philosophie de la boule postule une abondance absolue, et même une surabondance, première, une profusion, un excès ("Fülle »), et c'est cette abondance qui explique le réel, c'est face à sa complexité que le discours philosophique se construit, dans ses différences et ses contradictions.

En (9) enfin, il s'agit de montrer la manière dont le christianisme a repris le motif de la boule pour sa valeur œcuménique et divine.

Dans (7), (8) et (9), l'expression à l'infinitif ressaisit donc un changement de paradigme de pensée ou une manière de faire ou de penser qui actualise ou préfigure un tel changement. Cela va une nouvelle fois dans le sens de l'intuition de départ.

\section{Phrase entière}

Ce dernier cas fait presque exploser le tour syntaxique disloqué. L'indépendance du segment le plus à gauche est telle qu'on pourrait dire qu'il s'agit tout bonnement de deux énoncés distincts. Pourtant, des indices formels invitent d'emblée à considérer ce cas en continuité avec les analyses précédentes: la présence du tiret et la reprise.

(10) „Ihr seid gewissermaßen Götter und Söhne des Besten“- das ist es, was der Redner mit dem Radius, dem Dozentenstab, in dem sich die meisterlichen Rederechte kondensieren, in Erwiderung eines Arguments des linken Redners soeben zu erklären scheint. Begreift doch, Freunde, Verwandelte, Sphärengenossen, was diese Form da für jeden von uns bedeutet! (Sloterdijk 1999: 25-26)

(11) Wo bloße Umgebung war, soll die Kugel werden - mit diesem Imperativ setzt die Geometrie ins ethische Feld über. (Sloterdijk 1999: 51) 
(12) Interfaeces et urinam nascimur ${ }^{7}$, das ist nicht länger der antike Seufzer über die prekären physiologischen Anfänge des Menschenwerdens im mütterlichen Engpaß, das Wort wird jetzt zum anthropologischen Axiom und zur Parole in der Kampagne gegen die ungleichen Geburten. (Sloterdijk 2000: 72)

Un recours à la pragma-syntaxe permet de dépasser encore plus fermement l'apparente contradiction posée par ce type d'occurrences. On peut en effet envisager, avec Alain Berrendonner (2002), l'ensemble des occurrences étudiées comme des "périodes », afin d'en décrire un fonctionnement commun, le niveau rectionnel et le niveau topologique n'étant pas suffisants pour comprendre I'unité de cette configuration telle qu'elle se réalise en (10), (11) et (12). Dans la période, un état de la mémoire discursive $M$, qui prend corps dans une énonciation de clause $E$, va être modifié au fil du discours. Ces modifications sont de type praxéologique, c'est-à-dire qu'elles suivent une logique des actions, qui correspond bien souvent, dans les exemples étudiés par Berrendonner, à la mise en place d'un objet de discours ensuite déterminé, ou au contraire à des déterminations successives qui vont ensuite être mises en rapport avec un objet de discours, etc. La clôture de la période est signalée par l'accomplissement d'un acte énonciatif. Dans la configuration étudiée, un objet de discours, l'élément disloqué, est mis en place, puis déterminé dans l'énoncé d'accueil. Les segments antéposés en (10), (11) et (12) n'ont pragmatiquement aucune autonomie dans l'économie du texte. Ils consistent en des formules péremptoires, qui condensent une idée marquante déjà énoncée, de l'ordre d'un diagnostic critique décisif.

Les contradictions que soulève l'hétérogénéité des occurrences peuvent aussi être résolues, chez Sloterdijk, en les intégrant dans la problématique du discours autre. Le segment disloqué en (10) pastiche le texte du Nouveau Testament ( $\mathrm{Ihr}$ seid Götter und allzumal Kinder des Höchsten », Psaume 82: 6), l'hétérogénéité est montrée du doigt par les guillemets. (12) place à l'initiale, en italique dans le texte, une citation en latin attribuée à Saint Augustin. L'élément disloqué en (11) est un énoncé formé sur le moule d'une maxime présentée comme absolue. Disloquer participe ainsi chez Sloterdijk d'une mise en scène énonciative, polyphonique, qui peut être étendue à un très grand nombre des exemples analysés. La figure de l'oxymore en (4), l'adjectif « carlylisch » en (6), qui est une citation, les expressions à l'infinitif en (7), (8) et (9) - toutes ces occurrences ont pour point commun de convoquer d'autres énonciateurs, de faire entendre d'autres voix dans le texte.

Ces cas de prédication à un endroit censé se situer hors prédication donnent parfois lieu à des analyses en termes de « coups de force discursifs» (cf. FinkeLecaudey 1993 pour la prose de Nietzsche). L'auteur introduirait subrepticement des contenus controversés, en faisant par cette opération syntaxique passer du côté des présupposés tout ce qui est disloqué. Mais l'insertion dans le tissu textuel contredit quelque peu cette analyse, ou en tout cas, on ne peut s'en contenter. Pour notre analyse de la crise, il est certain que cette place est idéale pour poser un diagnostic de crise, que ce diagnostic soit établi de façon polémique ou non.

7. « Nous naissons entre les fèces et l'urine. » (Saint Augustin). 
Le philosophe déroule sa pensée à partir d'une situation critique ou complexe, placée à l'initiale de l'énoncé. L'initiale de phrase fait office de lieu de bilan et de moteur à la démonstration. Elle est également un lieu privilégié du discours autre, de mise en scène énonciative.

\section{La reprise dans l'énoncé d'accueil}

Soit la reprise est pronominale (par « das » souvent ou dans le cas de la dislocation d'un syntagme nominal par le pronom directement correspondant), soit elle se fait sous la forme d'une anaphore résomptive (aussi appelée conceptuelle ${ }^{8}$ ), introduite dans le corpus étudié majoritairement par dies- ${ }^{9}$. La DG permet un enchâssement spécifique des informations dans le texte: il y a continuité avec le co-texte gauche, mise en scène énonciative, mais aussi des apports rhématiques et énonciatifs qui vont maintenant être étudiés. Les anaphores pronominales seront laissées de côté.

\section{Anaphores résomptives et modalisation autonymique}

Les termes de reprise choisis par Sloterdijk sont souvent métalinguistiques (Satz, Operation, Formulierung, Redefigur...). Il est des énoncés qui savent décrire une situation à sa plus juste mesure - Sloterdijk n'a de cesse de se référer à une expression d'Elias Canetti et de la redonner dans son intégralité, dont deux fois de façon disloquée:

(13) Plötzlich alles schwarz von Menschen: Für jeden, dem die Sache der Emanzipationen, die Erhebung der Massen in den Subjektrang, am Herzen liegt, ereignet sich in dieser Formulierung ein peinlich nachhallender Affront. (Sloterdijk 2000: 13)

(14) Alles schwarz von Menschen - diese Redefigur gehört zu dem Zeitalter der Auflaufmassen, oder, wie man auch sagen könnte, der Versammlungs- und der Präsenzmassen, deren Charakteristikum darin besteht, daß große Zahlen von Menschen, Tausende, Zehntausende, Hunderttausende, im äußersten Fall Millionen, sich selbst als eine versammlungsfähige Größe erleben, indem sie an einem Ort, der sie alle faßt, zusammenströmen und in diesem massiven Konvent eine ungeheure Selbsterfahrung als wollendes, forderndes, wortergreifendes, gewaltemanierendes Kollektiv gewinnen. (Sloterdijk 2000: 15)

Le phénomène de réflexivité autonymique, tel que l'expose Jacqueline AuthierRevuz, apporte un éclairage intéressant sur les DG en (13) et (14):

8. L'anaphore conceptuelle condense par l'antécédent un contenu sémantique et dit quelque chose de ce contenu, alors que le pronom ne fait que le reprendre, sans jugement, ni évaluation ou commentaire.

9. Pour M.-H. Pérennec (2011), I'utilisation très fréquente du démonstratif plutôt que du défini dans l'anaphore résomptive s'explique par le « léger décalage sémantique » entre le lexème de reprise et le contenu repris. Selon W. De Mulder, il apparaît que « [I]es démonstratifs apportent toujours du nouveau: ils introduisent un nouveau référent ou un nouvel état du référent, ils changent le statut thématique du référent ou ils l'insèrent dans un nouvel univers » (De Mulder 1998: 30, cité par Kara / Wiederspiel 2011). Notons l'absence de dies- en (4). 
Dans tout fait de réflexivité autonymique, il y a un signe qui s'impose comme objet, propulsé sur le devant de la scène comme "personnage » auquel le dire fait référence, sortant par là de son rôle de rouage ordinaire de la machinerie du dire, voué à l'effacement dans l'accomplissement de sa fonction ordinaire de médiation. (Authier-Revuz 2002: 5)

C'est comme si la structure disloquée réalisait ce que décrit si bien AuthierRevuz: la DG fait sortir l'expression «Alles schwarz von Menschen » de «son rôle de rouage ordinaire de la machine du dire » et la « propulse sur le devant de la scène » (l'initiale de phrase). Elle est prise " dans sa matérialité singulière de signifiant et de signifié » (2002: 12) et ce deux fois dans le texte, par le même tour syntaxique! II n'y a pas de synonyme possible, les mots de Canetti sont là, dans leur " consistance et résistance », leur « irremplaçable singularité » (lbid.). La place du segment disloqué se révèle être la place de l'hétérogène, lieu privilégié d'accueil d'une séquence "prélevée telle quelle dans un autre lieu discursif » (2002: 14), en l'occurrence le texte de Canetti qui saisit poétiquement et puissamment le changement de paradigme, violent, que constitue l'apparition de la société de masse.

\section{«Résomption axiologisée » (Kara / Wiederspiel 2011)}

Dans la résomption axiologisée, il s'agit de " [r]éitérer du même tout en introduisant, dans le travail de conceptualisation, du nouveau qui relève de la manière de voir du scripteur " (Kara / Wiederspiel 2011). Ce concept est très intéressant par rapport à la thématique de la crise, parce que la situation posée comme critique à l'initiale de phrase va être soumise à deux traitements: d'abord un traitement axiologique, le scripteur va, dans la reprise, déjà évaluer, situer, catégoriser le contenu disloqué, puis un traitement prédicatif réalisé par le reste de l'énoncé d'accueil. Les termes de reprise peuvent aussi exprimer d'entrée de jeu le diagnostic critique sur la situation posée à l'initiale de phrase: Widerspruch (" contradiction »), Paradoxon (« paradoxe ») ou encore dans l'exemple (15), un groupe nominal métaphorique fortement teinté de subjectivité, dieser interaktive Krebs des Medienzeitalters (« ce cancer interactif de l'ère des médias »):

(15) Die dritte Verächtlichkeit des Menschen, seine Bloßstellung im System vulgarisierender, prostituierender und flexibilisierender Kommunikationen - dieser interaktive Krebs des Medienzeitalters ist für die Revolutionäre des 19. Jahrhunderts noch außer Sichtweite, nur einige eminente Künstler, Baudelaire und Mallarmé in erster Linie, haben auf die zunehmende Fixierung des Menschen in der Erniedrigung durch Trivialkommunikationen mit prophetischer Heftigkeit reagiert. (Sloterdijk 2000: 52)

Ce double niveau rend possible un « renouvellement partiel du thématique et du problématique, notamment en lui adjoignant une dimension interprétative » (Kara / Wiederspiel 2011 [en ligne]). La reprise introduit un commentaire actuel sur une situation déjà présentée de manière cinglante en dislocation (la communication de masse), mais qui ne fait pas encore partie de l'horizon du XIXe siècle, même si un petit nombre de penseurs visionnaires la pressentent déjà dans leurs œuvres. La DG rejoue en quelque sorte cette anticipation anachronique. 
On retrouve dans certaines analyses l'idée selon laquelle ce procédé relèverait d'un coup de force, non plus discursif mais interprétatif. Cependant, il faut se garder de généraliser et de considérer la DG comme lieu et place privilégiés d'un raisonnement fallacieux.

\section{Conclusion: figuralité de la DG}

Éric Bordas décrit la dislocation comme un « phénomène d'emphase énonciative » (1999: 224), caractéristique de la plume enthousiaste de Madame de Staël. On retrouve un même enthousiasme déclamatoire dans l'écriture de Peter Sloderdijk.

II n'est pas forcément pertinent d'envisager sa pratique de la DG comme un « coup de force interprétatif » ou " coup de force discursif ». II vaut mieux la voir comme une figure complexe intéressante: la DG est avant tout une forme rythmique dont Sloterdijk parvient très bien à exploiter les riches effets textuels.

On peut parler d'une "figuralité » ou d'un emploi figural de la DG. Selon Marc Bonhomme, les figures « apparaissent comme des phases de forte maîtrise du discours, où celui-ci s'auto-engendre à partir de structures programmées, où il s'exhibe dans son formalisme et où l'élocution se nourrit abondamment de la disposition » (2005: 66). Cette disposition sert bien chez Sloterdijk à poser ou ressaisir de façon prégnante des situations, des postures, des actions jugés par lui critiques, confirmant ainsi notre hypothèse de départ d'un rôle textuel essentiel de la DG dans le discours de la crise chez ce philosophe.

\section{Bibliographie}

\section{Fuvres de Peter Sloterdijk}

Sloterdijk, Peter (2000) Die Verachtung der Massen. Versuch über Kulturkämpfe in der modernen Gesellschaft. Frankfurt a.M.: Suhrkamp.

Sloterdijk, Peter (1999) Sphären II. Globen. Frankfurt a.M.: Suhrkamp.

\section{Références secondaires}

Authier-Revuz, Jacqueline (2002) Le Fait autonymique: Langage, Langue, Discours - Quelques repères. In: Jacqueline Authier-Revuz / Marianne Doury / Sandrine Reboul-Touré (éd.), Parler des mots - Le Fait autonymique en discours, Paris: Presses Sorbonne Nouvelle, p. 67-95.

Avanzi, Mathieu (2011) La Dislocation à gauche en français spontané. Étude instrumentale. [http://www2.unine.ch/repository/default/content/sites/ linguistique.francaise/files/shared/documents/Disloc-sujet-AM.pdf], dernière consultation le 24.10.2016.

Berrendonner, Alain (2002) « Les deux syntaxes ». Verbum XXIV / 1-2, p. 23-35. 
Blasco-Dulbecco, Mylène (1999) Les Dislocations en français contemporain. Étude syntaxique. Paris: Honoré Champion.

Bonhomme, Marc (2005) Pragmatique des figures du discours. Paris: Honoré Champion.

Bordas, Éric (1999) «Le style emphatique de Madame de Staël. Dislocation et extraction des "phrases" dans Corinne ou I'Italie ». In: Franck Neveu (dir.), Phrases: syntaxe, rythme, cohésion du texte, Paris: SEDES, p. 217-235.

Charolles, Michel / Péry-Woodley, Marie-Paule (dir.) (2005) Langue française 148, Les Adverbiaux cadratifs.

De Mulder, Walter (1998) « Du sens des démonstratifs à la construction d'univers ». Langue française 12, p. 21-32.

Finke-Lecaudey, Geneviève (1993) « Diskursfunktionen einiger topikalisierter Strukturen in Nietzsches Morgenröthe und Die fröhliche Wissenschaft ». In: Jean-François Marillier (éd.), Satzanfang - Satzende, Tübingen : Gunter Narr Verlag, p. 161-173.

Gardelle Laure (2010) « Dislocations à gauche et antépositions de compléments en anglais: formes, fonctionnalités et insertion en discours ». Discours 7, [https://discours.revues.org/7956], dernière consultation le 24.10.2016.

Kara, Mohamed / Wiederspiel, Brigitte (2011) «Anaphore résomptive conceptuelle et mémoire discursive: entre identité et altérité ». Itinéraires 2011 / 2, [https:// itineraires.revues.org/134?lang=fr\#text], dernière consultation le 26.11.2016.

Pérennec, Marie-Hélène (2001) « L'anaphore résomptive / conceptuelle en dies- ». Lylia 50, [http://langues.univ-lyon2.fr/medias/fichier/pe-rennec-mh-2011_1417600998957-pdf], dernière consultation le 26.11.2016.

Ricœur, Paul (1988) «La crise: un phénomène spécifiquement moderne? ». Revue de théologie et de philosophie 120, [http://fondsricoeur.fr/uploads/medias/ articles_pr/crise-4.pdf], dernière consultation le 08.03.2017, p. 1-19.

Schanen, François / Confais, Jean-Paul (2001) Grammaire de I'allemand. Formes et fonctions. Paris: Nathan Université.

Siblot, Paul (1998) "Variations sur un thème qui "toujours déjà" prédique ". Cahiers de praxématique 30, [https://praxematique.revues.org/2752], dernière consultation le 27.10.2016.

Sloterdijk, Peter (2003) Ni le soleil ni la mort. Jeu de piste sous forme de dialogues avec Hans-Jürgen Heinrichs. Trad. Olivier Mannoni. Paris: Fayard. 
